\title{
Optical properties of chromium-doped fluoroindate glasses
}

\author{
C. R. Mendonça, B. J. Costa, Y. Messaddeq,* and S. C. Zilio \\ Departamento de Física e Ciência dos Materials, Instituto de Física de São Carlos, Universidade de São Paulo, Caixa Postal 369, \\ 13560-970 São Carlos, SP, Brazil
}

(Received 16 October 1996; revised manuscript received 27 February 1997)

\begin{abstract}
This work reports on the optical properties of $\mathrm{Cr}^{3+}$ ions in the pseudoternary system $\mathrm{InF}_{3}-\mathrm{GdF}_{3}-\mathrm{GaF}_{3}$. Linear properties, investigated through absorption and emission spectra, provide information on the crystal field, the frequency, and number of phonons emitted during the absorption to the ${ }^{4} T_{2}$ band and the emission to the ${ }^{4} A_{2}$ ground state, and the Fano antiresonance line shape in the vicinity of the ${ }^{4} A_{2} \rightarrow{ }^{2} E$ transition. A study of the nonlinear refractive index as a function of the wavelength, carried out with the $Z$-scan technique, provides spectroscopic data about electronic transitions starting from the excited state. [S0163-1829(97)03129-9]
\end{abstract}

\section{INTRODUCTION}

Heavy-metal fluoride glasses have been extensively investigated due to their transparency in a wide spectral range, going from the ultraviolet to the infrared, which makes them suitable for the fabrication of optical fibers. ${ }^{1,2}$ Besides the ease of preparation, high concentrations of rare-earth and transition-metal ions can be incorporated into the matrix. These impurities present favorable spectroscopic properties for laser action, as reported in $\mathrm{Tm}^{3+}$-doped fluorozirconate fibers. ${ }^{3}$ From the viewpoint of basic research, a considerable effort has been devoted to characterize the optical properties of fluoride glasses doped with transition-metal ions. In particular, absorption spectra of $\mathrm{Cr}^{3+}$ have been investigated in several fluoride glasses, with emphasis placed on the study of ${ }^{4} A_{2} \rightarrow\left({ }^{2} E,{ }^{4} T_{2},{ }^{4} T_{1}\right)$ transitions. ${ }^{4-6}$ It was shown that the overlap of the broad ${ }^{4} T_{2}$ band and the sharp ${ }^{2} E$ and ${ }^{2} T_{1}$ lines gives rise to Fano antiresonances and a new approach for the assignment of the absorption bands in fluoride glasses was presented, with the determination of the crystal field and Racah parameters $(D q, B$, and $C){ }^{6}$ The values obtained were shown to be consistent with respect to the TanabeSugano diagram for ions in octahedral symmetry. On the other hand, in order to obtain good quality glasses one has to avoid devitrification processes. ${ }^{7}$ Recently, several compositions of the pseudoternary system $\mathrm{InF}_{3}-\mathrm{GdF}_{3}-\mathrm{GaF}_{3}$ with (20\% $\mathrm{ZnF}_{2}, 16 \% \mathrm{BaF}_{2}, 20 \% \mathrm{SrF}_{2}$, and $2 \% \mathrm{NaF}_{2}$ ) were investigated and they were shown to be stable concerning devitrification. ${ }^{8}$ In the present work, $\mathrm{Cr}^{3+}$ ions incorporated to these fluoroindate glasses have their optical properties characterized. The determination of linear properties was carried out through the measurement of absorption and emission spectra, and the results obtained turned out to be similar to those reported for BYZT and ZBLA glasses. ${ }^{6}$ However, we extended that previous work by performing an analysis of the ${ }^{4} A_{2} \rightarrow{ }^{4} T_{2}$ transition line shape, which gives the number and frequency of phonons emitted during the absorption process. The study of the Fano antiresonance in the vicinity of the ${ }^{4} A_{2} \rightarrow{ }^{2} E$ transition was also carried out, but the one related to the ${ }^{4} A_{2} \rightarrow{ }^{2} T_{1}$ transition could not be studied because it is barely observed. The emission spectrum of the ${ }^{4} T_{2} \rightarrow{ }^{4} A_{2}$ transition around $780 \mathrm{~nm}$ was measured and the mean lifetime of the excited state was found to be $250 \mu \mathrm{s}$. The emission line shape is also analyzed and the results are compared to the absorption case.

The analysis of nonlinear optical properties showed that chromium-doped glasses behave as Kerr media and the dependence of the nonlinear refractive index on the wavelength was investigated by means of the $Z$-scan technique. ${ }^{9}$ The results obtained indicate that contrary to chromium-doped oxide crystals, the charge-transfer band (CTB) located at the ultraviolet (UV) region does not contribute to the nonlinear index, but the transitions in the visible (VIS) region do. This result is in agreement with those of excited-state spectroscopy and four-wave mixing in fluoride crystals. ${ }^{10} \mathrm{~A}$ simple theoretical model was employed and spectroscopic data for electronic transitions starting from both the ground and excited states were determined.

\section{EXPERIMENTAL}

The samples used in this study have the following composition (in mol \%): $22 \mathrm{InF}_{3}, 2 \mathrm{GdF}_{3}, 18 \mathrm{GaF}_{3}, 20 \mathrm{ZnF}_{2}, 20$ $\mathrm{SrF}_{2}, 15.8 \mathrm{BaF}_{2}, 2 \mathrm{NaF}_{2}$, and $0.2 \mathrm{CrF}_{3} . \mathrm{InF}_{3}, \mathrm{GdF}_{3}$, and $\mathrm{CrF}_{3}$ were obtained by fluoration of $\mathrm{In}_{2} \mathrm{O}_{3}, \mathrm{Ga}_{2} \mathrm{O}_{3}$, and $\mathrm{Cr}_{2} \mathrm{O}_{3}$ with $\mathrm{NH}_{4} \mathrm{~F}$ and $\mathrm{HF}$, in a platinum crucible held at $400{ }^{\circ} \mathrm{C}$. All fluoride components were mixed and heated in a dry box under argon atmosphere at $700{ }^{\circ} \mathrm{C}$ for melting and $850^{\circ} \mathrm{C}$ for fining. ${ }^{11}$ The melt was then cast into a mold preheated to $280^{\circ} \mathrm{C}$ and slowly cooled down to room temperature. The samples obtained have good optical quality and are nonhygroscopic. It was found that concentrations of $\mathrm{CrF}_{3}$ above $0.5 \%$ enhances the devitrification process. The concentration that we used $(0.2 \%)$ corresponds to an average of $1.2 \times 10^{19} \mathrm{Cr}^{3+} / \mathrm{cm}^{3}$, but optical measurements have shown that the distribution of ions is not uniform. Absorption spectra in the UV-VIS region were obtained with a BOMEM DA8 Fourier transform spectrometer. Emission spectra were measured with a grating monochromator after pumping the sample with $488 \mathrm{~nm}$ light from an argon-ion laser. The lifetime of the fluorescence was determined by using a storage oscilloscope. All spectroscopic measurements were carried out at room temperature.

The optical nonlinearity was investigated by means of the 


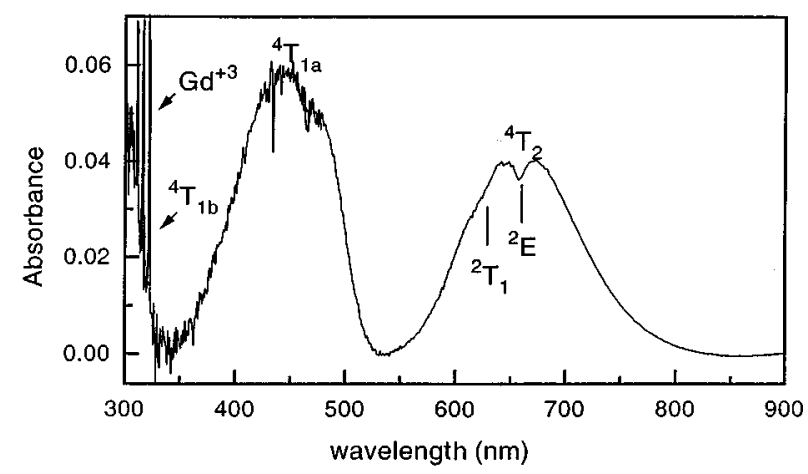

FIG. 1. Absorbance spectrum $\left(-\log _{10}\left(I / I_{0}\right)\right)$ of a 1.2-mm-thick sample of chromium-doped fluoroindate glass at room temperature. The transitions starting from the ${ }^{4} A_{2}$ ground state are indicated.

Z-scan technique introduced by Sheik-Bahae, Said, and Van Stryland, ${ }^{9}$ which is a powerful method of determining the nonlinear refractive index $n_{2}$ of a Kerr medium, a medium where the refractive index depends on the light intensity $I$, according to $n(I)=n_{0}+n_{2} I$. Basically, the method consists of translating a sample through the focus of a Gaussian beam and monitoring the changes in the far-field intensity pattern. The light field induces a $n_{2}$-dependent nonlinear phase and due to the transverse intensity profile of the Gaussian beam, the sample presents a lenslike effect. Therefore, it has the tendency of recollimating or defocusing the incident beam, depending on its $z$ position with respect to the focal plane. By monitoring the transmittance change through a small aperture placed at the far-field position, one is able to determine the induced nonlinear phase and hence the nonlinear refractive index. For the present measurements we have employed a time-resolved signal detection, which is suitable for slow response absorbers. ${ }^{12}$ As light sources we have used a krypton ion laser (413 nm), an argon-ion laser (476.5, 488, and $514.5 \mathrm{~nm}$ ), a dye laser operating with rodhamine 590 $(570-615 \mathrm{~nm})$ or DCM $(620-670 \mathrm{~nm})$ and a Ti:sapphire laser $(766 \mathrm{~nm})$.

\section{RESULTS AND DISCUSSION}

\section{A. Absorption spectrum}

Figure 1 shows the absorbance spectrum of a $1.2-\mathrm{mm}-$ thick sample of $\mathrm{Cr}^{3+}$-doped fluoroindate glass in the VIS region. Similarly to BYZT and ZBLA glasses two broadbands relative to the transitions ${ }^{4} A_{2} \rightarrow{ }^{4} T_{2}$ and ${ }^{4} A_{2} \rightarrow{ }^{4} T_{1 a}$ are observed around 660 and $440 \mathrm{~nm} .{ }^{6}$ A third band due to the transition ${ }^{4} A_{2} \rightarrow{ }^{4} T_{1 b}$ appears below $350 \mathrm{~nm}$, in addition to three sharp lines due to the $\mathrm{Gd}^{3+}$ absorption. Superposed to the ${ }^{4} T_{2}$ band two dips are observed, corresponding to Fano antiresonances due to the mixing with the ${ }^{2} E$ and ${ }^{2} T_{1}$ lines. By using the procedure described in Ref. 6 we found our spectrum consistent with respect to the TanabeSugano diagram for $\mathrm{Cr}^{3+}$ ions in octahedral symmetry, with crystal field and Racah parameter given by $D q=1547.7$ $\mathrm{cm}^{-1}, B=735.07 \mathrm{~cm}^{-1}$, and $C=3447.8 \mathrm{~cm}^{-1}$, resulting in $D q / B=2.11$. This value is essentially the same as BYZT and ZBLA, meaning that there are no substantial differences among the crystal fields of these glasses.
A line-shape analysis of the ${ }^{4} T_{2}$ band gives the number and frequency of the phonons emitted. Away from the sharp transition, the broadband can be fitted by the Pekarian form: ${ }^{13}$

$$
\alpha(\omega)=\alpha_{0} \frac{e^{-S} S^{p}}{\Gamma(p+1)},
$$

where $p=\left(\omega-\omega_{0}\right) / \Omega$ and $\alpha_{0}, S, \omega_{0}$, and $\Omega$ are adjustable parameters. $S$ is the mean number of phonons emitted in the transition and $\hbar \Omega$ their mean energy. The cutoff frequency $\omega_{0}$ corresponds to the longest wavelength observed in the spectrum such that the center of the band occurs at $\omega_{0}+\Omega$. The line shape of the ${ }^{4} A_{2} \rightarrow{ }^{4} T_{2}$ transition is fairly symmetrical, as expected for measurements carried out at room temperature. ${ }^{13}$ The fitting provided by Eq. (1) gives the phonon mean frequency $(\Omega)$ as $0.13 \times 10^{15} \mathrm{rad} / \mathrm{s}$, which corresponds to a wavelength of $15 \mu \mathrm{m}$. This is in agreement with the infrared absorption spectrum that presents a strong absorption for wavelengths longer than $10 \mu \mathrm{m}$. As discussed by Seeber et al., ${ }^{14} \Omega$ is associated with vibrations including the $\mathrm{Cr}^{3+}$ impurity, whose energy is less than the cutoff energy of the matrix glass as sensed by IR spectroscopy. The number of phonons emitted is of order 3 for the ${ }^{4} A_{2} \rightarrow{ }^{4} T_{2}$ transition, which is smaller than the order 5 for ruby, ${ }^{15}$ probably due to the weaker crystals field.

The study of the of the Fano antiresonance around $660 \mathrm{~nm}$ (mixing of ${ }^{4} T_{2}$ and ${ }^{2} E$ ) can be made according to the theory presented in Ref. 13. The antiresonance has its origin on the interference occurring between the phononless sharp transition with the $t_{2}{ }^{3}$ configuration and the broad transition to the $t_{2}{ }^{2} e$ configuration in which several phonons are emitted. When the absorption $\alpha_{B}(\omega)$ due to a broad vibronic band changes slowly in the vicinity of the sharp line, the total absorption coefficient $\alpha(\omega)$ is given by

$$
\alpha(\omega)=\alpha_{B}(\omega)+\alpha_{0}(\omega) \frac{\left(Q^{2}+2 \xi Q-1\right)}{\left(1+\xi^{2}\right)},
$$

where $\alpha_{0}(\omega)$ is proportional to $\alpha_{B}(\omega), \xi=\left(\omega-\omega_{r}\right) / \gamma$, with $\hbar \omega_{r}$ being the energy difference between the ground state and the sharp excited state modified by the interaction with the broad band, (1/2) $\gamma^{-1}$ is the lifetime of the sharp state against decay to the vibronic band ( $2 \hbar \gamma$ is the width of the sharp transition), and $Q^{2} \gamma$ is a measure of the strength of transitions to the modified sharp state, relative to the band. Figure 2 shows the Fano antiresonance with the background [given in Eq. (1)] already subtracted. The fitting to Eq. (1) gives the modified ${ }^{4} A_{2} \rightarrow{ }^{2} E$ transition frequency as $\omega_{r}=2.87 \times 10^{15} \mathrm{rad} / \mathrm{s}$, corresponding to a wavelength of $657.4 \mathrm{~nm}$. The value of $Q$ was found to be 0.08 , which is significantly smaller than the one obtained for the same transition in $\mathrm{MgF}_{2}: \mathrm{V}^{2+}{ }^{13}$ and the decay rate to the vibronic band $\left(\gamma=0.028 \times 10^{15} \mathrm{rad} / \mathrm{s}\right)$ yields the lifetime of the sharp state as 0.1 ps.

\section{B. Emission spectrum}

The emission spectrum at room temperature shown in Fig. 3 was obtained with a pumping wavelength of $488 \mathrm{~nm}$. A line-shape analysis with the Pekarian form as carried out for the absorption coefficient of the ${ }^{4} T_{2}$ band gives the phonon 


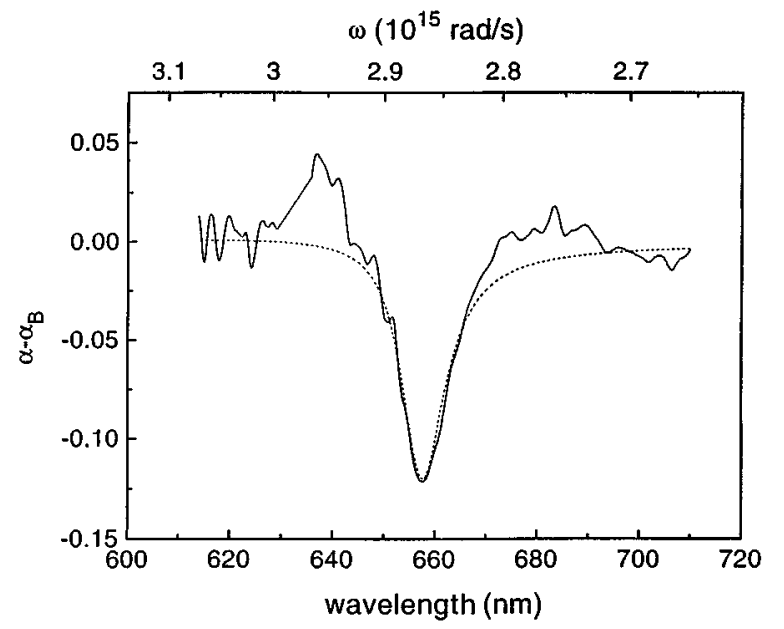

FIG. 2. ${ }^{4} A_{2} \rightarrow{ }^{2} E$ transition of $\mathrm{Cr}^{3+}$ in fluoroindate glass at room temperature. The dashed line represents the theoretical fitting given by Eq. (2) after subtraction of the background absorption.

frequency $(\Omega)$ as $0.11 \times 10^{15} \mathrm{rad} / \mathrm{s}$, corresponding to a wavelength of $18 \mu \mathrm{m}$. The number of phonons emitted is of order 2 for the ${ }^{4} T_{2} \rightarrow{ }^{4} A_{2}$ transition. These values are very close to those obtained for the absorption band. Owing to the glass disorder of the samples, the time evolution of the excitedstate population at room temperature, measured by monitoring the fluorescence decay with a storage oscilloscope, does not exactly follow an exponential, as expected for a threelevel system like ruby, but an average of several exponentials. This is due to random distribution of $\mathrm{Cr}^{3+}$ ions at nonequivalent positions inside the glass host, each one producing a slightly different time. Even though, an exponential was adjusted to the experimental data and as result, a mean lifetime around $250 \mu \mathrm{m}$ was obtained.

\section{NONLINEAR REFRACTIVE INDEX}

The normalized transmittance as a function of the sample position in a typical $Z$-scan measurement is shown in Fig. 4.

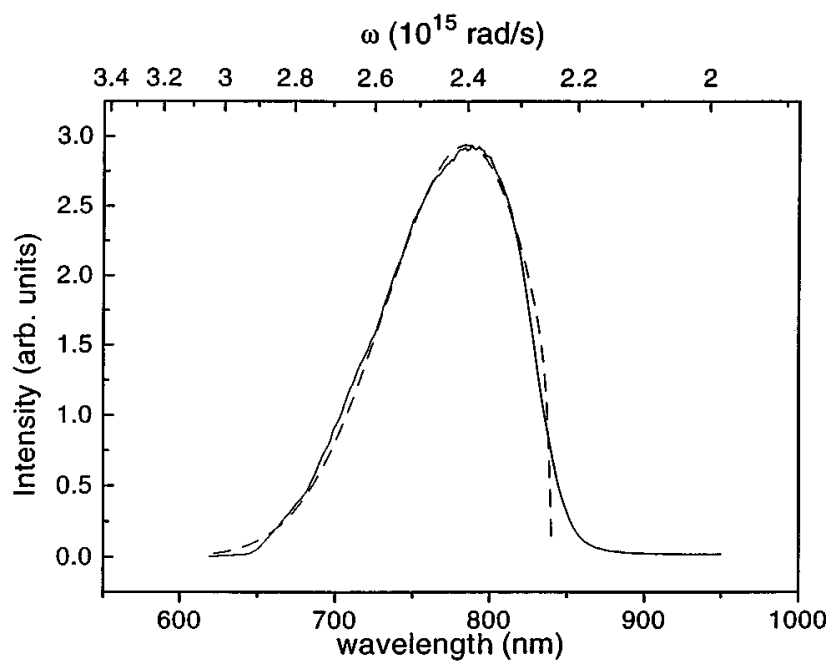

FIG. 3. Fluorescence of the $\mathrm{Cr}^{3+}$-doped fluoroindate glass at room temperature with a pumping wavelength of $488 \mathrm{~nm}$. The dashed line is the theoretical fitting provided by a Pekarian form with $S=2$ and $\Omega=0.11 \times 10^{15} \mathrm{rad} / \mathrm{s}$.

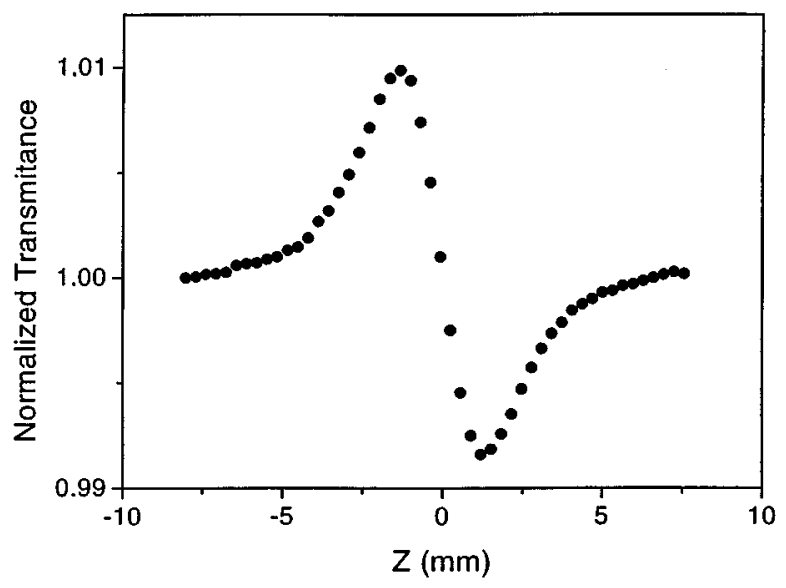

FIG. 4. $Z$ scan of a 1.2 -mm-thick sample at $608 \mathrm{~nm}, T=0.04$ and $I_{0}=58 \mathrm{~kW} / \mathrm{cm}^{2}$.

Since the peak occurs at $z<0$ we conclude that the nonlinearity is negative. ${ }^{16}$ For the particular curve shown we used $\lambda=608 \mathrm{~nm}$, an aperture with a transmittance $T=0.04$ and an intensity $I_{0}=58 \mathrm{~kW} / \mathrm{cm}^{2}$ at the center $(r=0)$ of the focal spot $(z=0)$ of the Gaussian laser beam. The nonlinear refractive index can be calculated from the transmittance difference between the peak and the valley according to ${ }^{9,16}$

$$
\Delta T_{p v}=(1-T)^{0.25} \frac{2 \pi}{\lambda} n_{2} I_{0} L,
$$

where $L$ is the sample thickness (1.2 $\mathrm{mm}$ in our experiment). By substituting the values used in the experiment we obtain $n_{2}=-6 \times 10^{-8} \mathrm{~cm}^{2} / \mathrm{kW}$ at $608 \mathrm{~nm}$. The magnitude of the nonlinear index depends on the electronic transitions involved and also on the concentration of chromium ions. We can get some useful information by normalizing $\left|n_{2}\right|$ to the absorption coefficient, which is also proportional to the concentration. For $514.5 \mathrm{~nm}$ we obtain $\left|n_{2}\right| / \alpha=1.6 \times 10^{-7}$ $\mathrm{cm}^{3} / \mathrm{kW}$, which is about two orders of magnitude smaller than the value obtained for a ruby sample with the phase conjugation technique at that wavelength. ${ }^{17}$ This indicates that the origin of the optical nonlinearity in chromium-doped fluoroindate glass must be different from that of ruby.

In order to verify the origin of the nonlinear index we have performed $Z$-scan measurements similar to those of Fig. 4 for several wavelengths. The values obtained for $\left|n_{2}\right|$ are shown as solid circles in Fig. 5, together with the absorption coefficient (full line) at the spectral region investigated. The results indicate that the variation of $\left|n_{2}\right|$ on the wavelength roughly resembles the absorption coefficient curve. When this fact occurs, the main contribution to the nonlinear index may come from the strong UV transition of $\mathrm{Cr}^{3+}$, known as charge transfer band (CTB), located typically around 200 $\mathrm{nm}$. These transitions were extensively investigated in several oxide hosts by excited-state spectroscopy and four-wave mixing. ${ }^{10}$ It is well established that in this case $n_{2}$ is positive, but in our measurements we obtained the opposite sign. Besides, results of excited-state spectroscopy of chromiumdoped fluoride crystals indicate the nonexistence of the CTB in the UV region, ${ }^{10}$ but only of a weak $3 d \rightarrow 4 s$ transition around $56000 \mathrm{~cm}^{-1}$ that could not explain our results because it would also yield a positive $n_{2}$. In this way we con- 


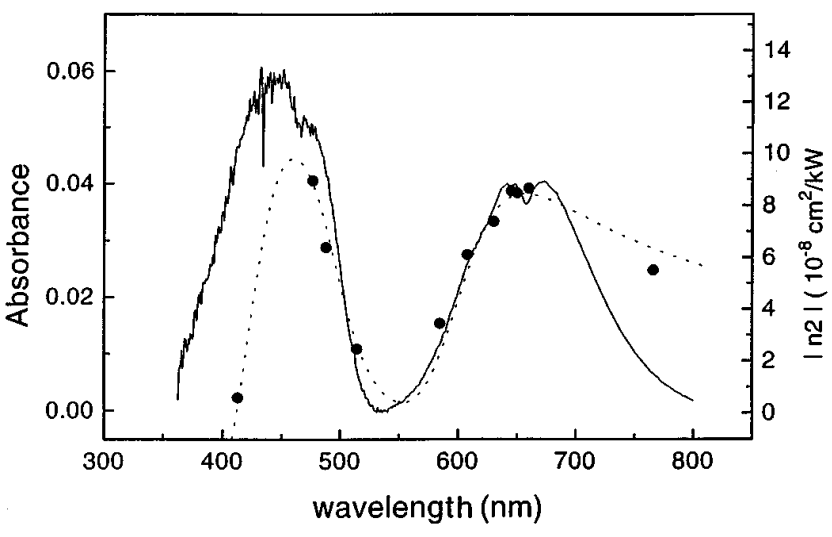

FIG. 5. Dependence of the nonlinear refractive index on the wavelength (solid circles), absorption coefficient (full line) and result of the theoretical fitting (dashed line). See text for details.

clude that no transition in the UV has any important effect in the optical nonlinearity. As the next step, we have to consider the possibility of a thermal origin for the nonlinearity. The sign of $d n / d T$ measured with a Pulfrich refractometer $\left(d n / d T \approx-10^{-6} /{ }^{\circ} \mathrm{C}\right)$ is negative and this could explain the sign of $n_{2}$, which for a thermal nonlinearity is proportional to $d n / d T$ times $\Delta T$. However, the temperature increase, $\Delta T$, depends on the total energy absorbed, which is proportional to $\alpha(\omega)$, times the energy mismatch between the absorbed and emitted photons. Therefore, the effect should be enhanced for blue photons and inhibited at the red side of the spectrum, but according to Fig. 5, the points at 413 and 766 $\mathrm{nm}$ have a behavior markedly opposite to this. Moreover, the rise time of the aperture transmittance does not depend on the laser spot size, which is inconsistent with a thermal effect ( $\tau_{t h}$ proportional to $\omega_{0}{ }^{2}$ ) and thus, the thermal origin for the optical nonlinearity has to be ruled out.

The above analysis lead us to the conclusion that the only possible explanation for the origin of the nonlinear refractive index has to be related to electronic transitions occurring in the VIS region. In order to confirm this assumption we have employed a model where $n_{2}$ is proportional to the susceptibility difference between the ground and excited states. ${ }^{18}$ According to the model presented in Ref. 19, the value of $n_{2}$ as a function of the light frequency $\nu$ is given as

$$
n_{2}(\nu)=\frac{\alpha(\nu) \tau c}{8 \pi^{2} h \nu^{2}}\left(\sum_{i \neq 1} \frac{\sigma_{l i}{ }^{(0)} \Delta_{l i}}{1+\Delta_{l i}{ }^{2}}-\sum_{i \neq 0} \frac{\sigma_{0 i}{ }^{(0)} \Delta_{0 i}}{1+\Delta_{0 i}{ }^{2}}\right)
$$

where $\tau$ is the excited-state lifetime $(250 \mu \mathrm{s}), \sigma_{0 i}{ }^{(0)}$ and $\sigma_{1 i}{ }^{(0)}$ are, respectively, the absorption cross sections for transitions starting from the ground state (0) and the first excited state (1), which corresponds to the ${ }^{4} T_{2}$ band, and $\Delta_{0 i}$ and $\Delta_{1 i}$ are adimensional detunings defined as

$$
\begin{gathered}
\Delta_{0 i}=\frac{\nu_{i}^{2}-\nu^{2}}{\gamma_{0 i} \nu}, \\
\Delta_{1 i}=\frac{\left(\nu_{i}-\nu_{1}\right)^{2}-\nu^{2}}{\gamma_{1 i} \nu},
\end{gathered}
$$

where $h \nu_{i}$ is the energy of the $i$ th level, and $\gamma_{0 i}$ and $\gamma_{1 i}$ are linewidths of transitions starting, respectively, from the ground and excited states and ending at the $i$ th level. The two terms inside parenthesis in Eq. (4) are, respectively, related to the susceptibilities of the excited and ground states. Except for the absolute values of the absorption cross sections, all parameters related to the ground state can be extracted from the absorption spectrum. The parameters related to the excited state and the cross-section absolute values of the ground state can be found by fitting the experimental data to Eqs. (4) and (5). The result is given by the dashed line of Fig. 5 and the spectroscopic parameters obtained through the fitting procedure are presented in Table I. Since there are several adjustable parameters, the values obtained have an uncertainty of about $20 \%$.

The cross-section absolute values for transitions starting from the ground state, obtained with the model described above, agrees with the absorption coefficient measured $[\alpha(\nu)=\mathbf{N} \sigma(\nu)]$. For the ${ }^{4} A_{2} \rightarrow{ }^{4} T_{2}$ transition, for instance, we have $\alpha \approx 1 \mathrm{~cm}^{-1}$, corresponding to $\sigma \approx 0.8 \times 10^{-19}$ $\mathrm{cm}^{2}$, which is about one half of the value presented in Table I. Therefore, the model predicts, within the experimental error, the same order of magnitude for the cross section measured by absorption spectroscopy and the discrepancy of the factor 2, which is not unusual in this sort of measurement, could be related to the nonuniformity of the $\mathrm{Cr}^{+3}$ distribution. The electronic transitions presented in Table I are interconfigurational, except for the ${ }^{4} T_{2} \rightarrow{ }^{4} T_{1 a}$ transition, which is intraconfigurational. Accordingly, its cross section is about two orders of magnitude smaller than the others.

\section{CONCLUSIONS}

In summary, we have investigated both linear and nonlinear optical properties of the chromium-doped fluoroindate glass. The visible absorption spectrum is quite similar to other fluoride glasses doped with $\mathrm{Cr}^{3+}$. Emphasis was placed on the study of the parameters that characterize the broad ${ }^{4} T_{2}$ absorption and the Fano antiresonance line shapes. By measuring the fluorescence we obtained the

TABLE I. Spectroscopic parameters for the electronics transitions of $\mathrm{Cr}^{3+}$ in fluoroindate glass.

\begin{tabular}{lccccc}
\hline \hline Transitions & ${ }^{4} A_{2}\left(t_{2}{ }^{3}\right) \rightarrow$ & ${ }^{4} A_{2}\left(t_{2}{ }^{3}\right) \rightarrow$ & ${ }^{4} A_{2}\left(t_{2}{ }^{3}\right) \rightarrow$ & ${ }^{4} T_{2}\left(t_{2}{ }^{2} e\right) \rightarrow$ & ${ }^{4} T_{2}\left(t_{2}{ }^{2} e\right) \rightarrow$ \\
& ${ }^{4} T_{2}\left(t_{2}{ }^{2} e\right)$ & ${ }^{4} T_{1 a}\left(t_{2}{ }^{2} e\right)$ & ${ }^{4} T_{1 b}\left(t_{2} e^{2}\right)$ & ${ }^{4} T_{1 a}\left(t_{2}{ }^{2} e\right)$ & ${ }^{4} T_{1 b}\left(t_{2} e^{2}\right)$ \\
\hline$\omega\left(10^{14} \mathrm{~Hz}\right), \lambda(\mathrm{nm})$ & $4.7(639)$ & $6.9(435)$ & $1.1(275)$ & & \\
$\sigma\left(10^{-19} \mathrm{~cm}^{2}\right)$ & 1.8 & 2.9 & 3.6 & $<0.03$ & 0.9 \\
$\gamma\left(10^{14} \mathrm{~Hz}\right)$ & 1.0 & 1.5 & 2.0 & 1.0 & 1.0 \\
\hline \hline
\end{tabular}


excited-state lifetime, and the number and frequency of phonons emitted during the decay to the ground state. The analysis of the nonlinear refractive index as a function of the wavelength led us to the conclusion that its origin is related to electronic transitions in the VIS range. The fitting of the experimental data to the theoretical model provided spectroscopic parameters for both the ground and excited states.

\section{ACKNOWLEDGMENTS}

This research was supported by Fundação de Amparo à Pesquisa do Estado de São Paulo (FAPESP) under Grant No. 95/4203-3 and the program PRONEX, Grant No. 4.1.96.0935.00. The authors thank V. Pilla for her help in part of the measurements.

${ }^{9}$ M. Sheik-Bahae, A. A. Said, and E. W. Van Stryland, Opt. Lett. 14, 995 (1989).

${ }^{10}$ S. C. Weaver and S. A. Payne, Phys. Rev. B 40, 10727 (1989).

${ }^{11}$ Y. Messaddeq, A. Delben, M. Broscolo, M. A. Aegerter, A. Soufiane, and M. Poulain, J. Non-Cryst. Solids 161, 210 (1993).

${ }^{12}$ L. C. Oliveira and S. C. Zilio, Appl. Phys. Lett. 67, 2121 (1994).

${ }^{13}$ M. D. Sturge, H. J. Guggenheim, and M. H. L. Pryce, Phys. Rev. B 2, 2459 (1970).

${ }^{14}$ W. Seeber, E. A. Downing, L. Hesslink, M. M. Fejer, and D. Ehrt, J. Non-Cryst. Solids 189, 218 (1995).

${ }^{15}$ D. S. McClure, J. Chem. Phys. 36, 2757 (1962).

${ }^{16}$ M. Sheik-Bahae, A. A. Said, T. Wei, D. J. Hagan, and E. W. Van Stryland, IEEE J. Quantum Electron. QE-26, 760 (1990).

${ }^{17}$ T. Catunda and J. C. Castro, Opt. Commun. 63, 185 (1987).

${ }^{18}$ H. L. Fragnito, S. F. Pereira, and A. Kiel, J. Opt. Soc. Am. B 4, 1309 (1987).

${ }^{19}$ E. A. Gouveia, I. Guedes, J. C. Castro, and S. C. Zilio, Phys. Rev. B 46, 14387 (1992). 